\title{
CHARACTERIZATION OF SOME ELEMENTARY TRANSFORMATIONS
}

\author{
CHIN-SHUI HSÜ
}

1. Introduction. The purpose of this paper is to study some elementary transformations of surfaces embedded in a 3-dimensional Euclidean space $E^{3}$. This will be developed analogously to the following theorem [1]:

Translation Theorem. Given two closed orientable surfaces $S, \bar{S}$ and a homeomorphism $h: S \rightarrow \bar{S}$ such that: (1) each line joining corresponding points is parallel to a fixed direction $E$, (2) the mean curvatures at corresponding points are equal; moreover $S, \bar{S}$ are assumed not to contain pieces of cylinders in E-direction. Then $h$ is a translation.

All surfaces mentioned will be of class $C^{2}$. The notations in [1] will be adopted except that German letters will be replaced by corresponding capital English ones. For example: $X, N, H$ and $d A$ are respectively used to denote the position vector, the unit vector along inward normal direction, the mean curvature and the surface element of a surface $S$. When a second surface $\bar{S}$ is mentioned, the corresponding quantities are represented by the same letters with bars above them. As in [1], the following formulas will be used:

$$
\begin{aligned}
& d X \times d X=2 N d A, \\
& d X \times d N=-2 H N d A .
\end{aligned}
$$

A closed nonself-intersecting surface $S$ is said to be convex with respect to a given point $0,(1)$ if every straight line through 0 meets $S$ at no point, at one point of contact or at two distinct points, (2) if there is a differentiable homeomorphism $f: S \rightarrow S$ such that each straight line joining corresponding points passes through 0 .

We intend to prove the following theorems:

THEOREM 1. Given two closed orientable surfaces $S, \bar{S}$ and a differentiable homeomorphism $h: S \rightarrow \bar{S}$ such that: (1) each straight line $P \bar{P}$ joining the corresponding points $P$ and $\bar{P}$ passes through a fixed point 0 ; (2) with 0 as origin, the quantities $X, \bar{X}, H, \bar{H}$ are related to each other either by (i) $\bar{H} \bar{X}=H X$ throughout $S$ and $\bar{S}$ or by (ii) $\bar{H} \bar{X}=-H X$ throughout $S$ and $\bar{S}$. Moreover $S, \bar{S}$ are assumed not to contain pieces of cones with vertex 0 . Then $h$ is a homothetic transformation with center 0 and with a

Received by the editors August 18, 1958. 
positive or negative constant of proportionality according as (i) or (ii) holds.

THEOREM 2. Given two closed orientable surfaces $S, \bar{S}$ and a differentiable homeomorphism $h: S \rightarrow \bar{S}$, such that: (1) each segment $P \bar{P}$ joining the corresponding points $P$ and $\bar{P}$ subtends a constant angle $P 0 \bar{P}$ about $a$ fixed point 0 , (2) with 0 as origin, $H X$ and $\bar{H} \bar{X}$ are equal in magnitude. Moreover, $S, \bar{S}$ are assumed not to contain pieces of cones with vertex 0 . Then $h$ is a similarity with 0 as center of similitude.

THEOREM 3. Given two closed orientable surfaces $S, \bar{S}$ and a differentiable homeomorphism $h: S \rightarrow \bar{S}$ such that: (1) each straight line $P \bar{P}$ joining corresponding points $P$ and $\bar{P}$ passes through a fixed point $0 ;(2)$ with 0 as origin, the quantities $X, \bar{X}, H, \bar{H}$ are related to each other either by (i) $\bar{H} \bar{X}=-(H+2 X \cdot N / X \cdot X) X$ throughout $S$ and $\bar{S}$; or by (ii) $\bar{H} \bar{X}$ $=(H+2 X \cdot N / X \cdot X) X$ throughout $S$ and $\bar{S}$. Moreover $S, \bar{S}$ are assumed not to contain either pieces of cones with vertex 0 or the point 0 itself. Then $h$ is an inversion with center 0 and with real or pure imaginary radius of inversion according as (i) or (ii) holds.

I am grateful to Professor T. K. Pan for his encouragement during the preparation of this paper.

\section{Proofs of theorems.}

Proof of Theorem 1. Assume $\bar{H} \bar{X}=H X$. Write $\bar{X}=k X$, where $k=H / \bar{H}$.

CASE 1. $0 \notin S$ and $0 \notin \bar{S}$. Then $k \neq 0, \infty$.

$$
\begin{aligned}
d \bar{X} \times d \bar{X} & =(k d X+X d k) \times(k d X+X d k) \\
& =k^{2}(d X \times d X)+2 k(X d k \times d X) .
\end{aligned}
$$

By (1.1), we have

$$
\bar{N} d \bar{A}=k^{2} N d A+k(X d k \times d X),
$$

whose scalar product with $\bar{H} \bar{X}(=H X)$ gives

$$
(\bar{X} \cdot \bar{N}) \bar{H} d \bar{A}=k^{2}(X \cdot N) H d A .
$$

Let $a=(N \times X) \cdot d X, b=(\bar{N} \times X) \cdot d X$ and note that $d d X=0$, then by use of (1.1) and (1.2), we obtain

$$
\begin{aligned}
d a & =2(X \cdot N) H d A+2 d A, \\
d b & =-X \cdot(d \bar{N} \times d X)+2(\bar{N} \cdot N) d A \\
& =\left(2 / k^{2}\right)(\bar{X} \cdot \bar{N}) \bar{H} d \bar{A}+2(\bar{N} \cdot N) d A
\end{aligned}
$$

since 


$$
\bar{X} \cdot(d \bar{N} \times d \bar{X})=k^{2} X \cdot(d \bar{N} \times d X) .
$$

Hence, by (2.1) we have

$$
d(a-b) / 2=(1-\bar{N} \cdot N) d A .
$$

From Stokes' Theorem, it is evident that

$$
\iint_{S}(1-\bar{N} \cdot N) d A=0 .
$$

Since $1-\bar{N} \cdot N \geqq 0$, and $d A$ always keeps the same sign, we have

$$
1-\bar{N} \cdot N=0
$$

and therefore

$$
N=\bar{N} \text {. }
$$

Moreover, since $\bar{N} \cdot d \bar{X}=0, N \cdot d X=0$, we have

$$
(N \cdot X) d k=0 .
$$

Hence $k=$ constant, unless $N \cdot X=0$.

Let $R$ be the set of points of $S$ at which $N \cdot X=0$. Then every point of $R$ (if there is any) is not an interior point; for otherwise, $S$ would contain a piece of cone with vertex 0 . Hence every point of $R$ is a limiting point of $S-R$, and, due to the continuity of $k=H / \bar{H}$, $k=$ constant throughout $S$. Moreover $N=\bar{N}$ implies that $k$ is positive. Consequently $h$ is a homothetic transformation with center 0 and with positive constant of proportionality.

CASE 2. $0 \in S$ or $0 \in \bar{S}$. Without loss of generality, we may assume $0 \in S$. In any open set $U$ of $S$ containing 0 , take a neighborhood $V$ of 0 . Let $V^{\prime}$ be the boundary of $V$ (and so also of $S-V$ ). Since $(1-\bar{N} \cdot N$ ) $d A$ always keeps the same sign

$$
\left|\iint_{S-U}(1-\bar{N} \cdot N) d A\right| \leqq\left|\iint_{S-V}(1-\bar{N} \cdot N) d A\right| .
$$

The expression on the right of (2.3) is equal to

$$
\frac{1}{2}\left|\int_{V^{\prime}}[(N-\bar{N}) \times X] \cdot d X\right|
$$

because of (2.2) and Stokes' Theorem, and it can be made as small as we please by choosing $V$ small enough, while the expression on the left of (2.3) remains fixed. Hence

$$
\iint_{S-U}(1-\bar{N} \cdot N) d A=0
$$


Following the same argument as in Case 1, we have $k=$ positive constant in $S-U$ for every open set $U$ of $S$ containing 0 . Hence $k=$ positive constant throughout $S$, since $k$ is continuous.

Assume $\bar{H} \bar{X}=-H X$. Write $\bar{X}=-k X$ where $k=H / \bar{H}$. Through similar arguments as above, we obtain

$$
\iint_{S}(1+\bar{N} \cdot N) d A=0,
$$

which gives $\bar{N}=-N$ and therefore $k=$ positive constant.

REMARK 1. Theorem 1 still holds when $S$ and $\bar{S}$ are not closed but bounded with boundaries $B$ and $\bar{B}$, such that $h(B)=\bar{B}$ and at corresponding points on $B$ and $\bar{B}$, we have $\bar{N}=N$ for case (i) or $\bar{N}=-N$ for case (ii). This is evident, because

$$
\begin{array}{ll}
\iint_{S}(1-\bar{N} \cdot N) d A=\frac{1}{2} \int_{B}[(N-\bar{N}) \times X] \cdot d X & \text { for case (i), } \\
\iint_{S}(1+\bar{N} \cdot N) d A=\frac{1}{2} \int_{B}[(\bar{N}+N) \times X] \cdot d X & \text { for case (ii). }
\end{array}
$$

REMARK 2. If we consider the more general condition $H X=r \bar{H} \bar{X}$, where $r$ is a constant, without loss of generality, we may assume $|r| \leqq 1$. Then instead of (2.2) we get

$$
\frac{1}{2} d(a-r b)=(1-r N \cdot \bar{N}) d A .
$$

Hence

$$
\iint_{S}(1-r \bar{N} \cdot N) d A=0 .
$$

This equation implies

$$
1-r N \cdot \bar{N}=0
$$

which is impossible unless $r= \pm 1$.

Corollary. Given a closed orientable surface $S$ convex with respect to a fixed point 0 . With 0 as origin, the quantities $X, H, X^{\prime}, H^{\prime}$ at points corresponding under $f$ are related to each other by $H^{\prime} X^{\prime}=-H X$. Then $S$ is symmetric with respect to 0 .

Proof. It is clear that $f: S \rightarrow S$ satisfies the assumptions in Theorem 1. Hence it is a homothetic transformation with center 0 and with negative constant of proportionality $-k$. Since both $P P^{\prime}$ and $P^{\prime}\left(P^{\prime}\right)^{\prime}$ 
pass through $0,\left(P^{\prime}\right)^{\prime}$ should be either $P$ or $P^{\prime}$, and since $f$ is one-one, $\left(P^{\prime}\right)^{\prime}=P$. Hence $k^{2}=1$, and $k=1$. Therefore $S$ is symmetric with respect to 0 .

Proof of Theorem 2. There is a transformation $g$ in $E^{3}$ (which is either a single rotation about an axis through 0 or such a rotation followed by a reflection against a plane through 0 ), such that each straight line $0 P$ is transformed into $0 \bar{P}$ where $P, \bar{P}$ are points corresponding under $h$.

Let $S^{*}=g(S)$. It is clear that $h g^{-1}: S^{*} \rightarrow \bar{S}$ satisfies the assumptions of Theorem 1, and hence is a homothetic transformation with center 0 . Therefore $h$ is a similarity.

Proof of Theorem 3. Let $g$ be the inversion about the unit sphere with center 0 . Denote by $X^{*}, H^{*}$ and $N^{*}$ the position vector, the mean curvature and the unit vector along inward normal direction at $P^{*}=g(P)$ of $S^{*}=g(S)$, respectively. By simple calculations we obtain

$$
H^{*} X^{*}=-\left(H+2 \frac{X \cdot N}{X \cdot X}\right) X,
$$

which reduces to $H^{*} X^{*}=\bar{H} \bar{X}$ for case (i) and to $H^{*} X^{*}=-H X$ for case (ii). Hence $h g^{-1}: S^{*} \rightarrow \bar{S}$ is a homothetic transformation with center 0 and with positive or negative constant of proportionality according as (i) or (ii) holds. Thus $h=\left(h g^{-1}\right) g$ is an inversion about 0 , and the radius of inversion is real or pure imaginary according as (i) or (ii) holds.

REMARK. Theorem 3 still holds when $S$ and $\bar{S}$ are not closed but bounded with boundaries $B$ and $\bar{B}$ such that $h(B)=\bar{B}$ and at corresponding points on the boundaries $\bar{N}=-N+2(X \cdot N / X \cdot X) X$ for case (i) or $\bar{N}=N-2(X \cdot N / X \cdot X) X$ for case (ii). This is evident because $N^{*}=-N+2(X \cdot N / X \cdot X) X$.

COROLlaRy. If $S$ is a closed orientable surface convex with respect to a point 0 not on $S$, and with 0 as origin we have $H=-(X \cdot N / X \cdot X) X$. Then $S$ is a sphere with center 0 .

Proof. Since $H X=-(H+2(X \cdot N / X \cdot X)) X$, each point of $S$ is invariant under the inversion about a sphere with center 0 and with real radius. Consequently, $S$ itself is a sphere with center 0 .

\section{REFERENCE}

1. H. Hopf and K. Ross, Ein Satz aus der Flächentheorie im Grossen, Arch. Math. vol. 3 (1952) pp. 187-192.

National Taiwan University, Taiwan, China 